\title{
Erhu Concerto in 1990s
}

\author{
Jindi Zhang
}

Art College, Shandong University, Weihai, 264209, China

\section{Keywords: Erhu concerto, Art}

\begin{abstract}
After 1980s, the west modern music flood into China, producing a shock to Chinese music industry and resulted in very profound impact on the development of national music. In the late 1990s, the impact of pop music also had a great impact on erhu music creation and the music creation in the later period had pop colors. There were about 50 concerto works in this period including "Looking into the Wildness", "Royal Love", "Lady Yang's story", "Goddess Marriage Fantasia", "Autumn"; the paper will choose "First Erhu Rhapsody" "Rivers and Cloud Dream" for analysis and commentary.
\end{abstract}

\section{Artistic characteristics of erhu concerto}

\section{Erhu skills and sound expansion}

Looking the works of 1990s from playing skills and creative ideas, the works catch the element characteristic of national tone, change into a particular semantic style, and the pursuit of difficult skills were complicated changes, the audience for these tonal elements, you can sense and cannot be found, familiar and a sense of novelty. Ultra-hard technology is one of the primary factors in the pursuit of satisfaction because virtuoso became the young generation musicians seeking skills favorites.

Intonation and rhythm skills due skill works highlight the personality, become difficult in the difficult technical problems, each composer when using the beat, the rhythm of change, and the use of artificial mode, are in accordance with the individual needs of the author's own design. This is a player who, for different composers must have a new understanding and work skills to master the process of re-training. This is the traditional tuning there is a pattern comparison, the player put a lot more time on skill training, while ignoring the artistic value of the work itself.

The artificial mode use in the works, a lot of lifting sound appears, the novel tuning further increases the difficulty of intonation skills.

The rapid decorative changing sound group is another challenge for the accuracy of intonation. Large span of intervals jumped more and more difficult, faster and faster. Use diminished chords, diminished seventh chords broken chords of new skills.

The frequent tempo change goes beyond the change of pace, disrupting the law stress; enhance freedom and irregular rhythms performed.

The new technique uses some special national characteristics simulation works, such as: bow stick hits tube, hand slaps tube, skip and pause bow new bow method, etc.

\section{Integration of Western compositional techniques and Chinese Aesthetics}

Through half a century of development of erhu playing skills, there has trained a large number of high-tech standards of player till 1990s. The players' requirement for new erhu new works is also increasing. "First Erhu Rhapsody" was created by the author Mr. Wang Jianmin after received the solicitation from the performer Deng Jiandong. The works required that: higher degree of difficulty, newer language and heavier weight.

The composition starts from the A tone in treble, around A tone introduces opening section, clever ornaments, fell sound end of the sentence, the people of the world into the dawn blurred, mysterious mountain scenery, quiet seems blowing looming song.

Andante - a little active - turn to adagio, the fixed and constant bass is like Dongzutaige in a multi-voice and sing, sing all sprout, A fine singing jumping fun. Singing melodies like Philco, like Songs, like Yi songs are like and do not like, and feel not catch; this is the most special thing works. On the use of the Miao Songs tone elements, the size of the house above the three-octave voice 
coexist diacritical color as music clips, using the twelve-tone composition technique to develop a new set of artificial pentatonic tuning nine, became music style The foundation, which produces melodic tone color changing effects. While maintaining the use of the original multi-ethnic folk songs, fly three octave model, the hearing still feel song features, but a new tone sound different. Music generated a lot of changes in tone, but has not caused the West tuning chromatic effects, but to the rich ethnic flavor adds mystery. Artificial mode with three degrees of the unique combination of stacked plastic to expand the use of acoustic space, the use of modern harmonies naturally produces a flexible and ethereal sound.

The author used the approach of irregular rhythm of Western modern music, applying a multi-ethnic transformation uses a variety of beat including Yi five-beat rhythm and Dai three-beat. And seven beat, six beat, five beat, four beat, three beat, two beat etc., clever and frequent use of the beat rhythm changes, which combines the use of multi-ethnic beat, irregular beats stress dislocation caused by the development of music driving force.

Before the composition enters into climax, it uses two special playing techniques, which riches sound. Erhu bow sticks the tube, like bamboo pole dance warm percussion rhythm. Hand hits the leather of cube imitates the people were clapping, stamping feet, slapping carnival percussion sound emitted, like a carnival prelude to gradually intensive plucked, flop the song to a climax.

\section{Forms of works and contents' artistry, literariness characteristics}

1). Pursue personality and break tradition

2). China and west compatibility, describe shape and emotion

\section{Creation of erhu concerto and development of performing arts}

In 1991, held the fourteenth session of the "Spring of Shanghai" Erhu Competition (Dunhuang cup); in 1993, held "to commemorate the 100th anniversary of national musician Hua Yanjun" National Invitational Nanjing National Instrumental Music; in 1995, China hosted 95 international instrumental Performance of "Futura Cup" International Chinese Ethnic Music Competition; "Tianhua Cup" National Youth erhu competition; in 1999, "Tianhua Cup" National Youth erhu game. In 1990s, national instrumental scale events, the game tracks requires rise to a higher technical specifications, which contributed to the emergence of new high-tech works, like "First Erhu Rhapsody" is the launch of a new work in the competition.

The author successfully uses the communion and folk characteristics of rhapsody structure and traditional Chinese plate structure, and builds up a new framework. And groundbreaking use of artificial mode both retained the main elements of material can reconstruct the features of traditional and novel materials to construct a new thematic material, combined with the new framework to construct an overall framework. "A mad" communion under output in Eastern and Western cultures, like a half-breed, which integrate the communion elements Chinese culture and Western culture, the formation of a new life form. But the acceptance of this new work is also still taken some time and an opportunity. "First Erhu Rhapsody" participated and won the 1988 sixth national music works (including ethnic instrumental solos, ensemble) ten years contest prize (first prize vacancies) after the remained dormant, variety in the 90's game tournaments and did not use "First Erhu Rhapsody", "First Erhu Concerto" and "Rivers and Cloud Dream" This highly difficult work, "Spring of Shanghai" Erhu Competition specify "Sanmenxia Capriccio" in 1991 as the final track, which compared 1985 Beijing Erhu Invitational specified track "Great Wall Capriccio" is obviously technically and art are showing but not into the back of the phenomenon. This phenomenon is explained on the one hand the difficulties of the work was far beyond the level of the erhu technology, explains the aesthetic ideas of professionals and other music industry players remain in the traditional arts Viewpoint more, the new musical language of Acceptance and take some time to digest grasp. Then on the one hand, by the large climate impact of the market economy and the impact of popular music, the music of this period led to the popularity, popularity, popularity direction.

The development of erhu art needs works in many forms and styles, but also needs works with highly different technologies. "First Erhu Rhapsody" as a virtuoso type of new works, to expand the 
power of artistic expression erhu played a role in promoting reform and innovation as the composer of new ideas, but also set a good example for the later. But after "First Erhu Rhapsody" and other large and challenging works constantly, the pursuit of the technical difficulty of the erhu is much higher than the pursuit of erhu art itself and the development trend of the phenomenon caused concern and reflection of the music industry. Difficult is the standard technology, not art standards, so when organizing the Music Competition of the highly artistic rather than technical in the first judgment, in order to guide the new force to a healthy direction. Meanwhile, the guide on the art of teaching should also be placed on the top position, and not just the culture of the game to players. The song is not the size and difficulty, but rather spread wide and far. Music requires listening, requires audience interaction and resonance, the song "difficult" and few, not the audience's musical vitality can go far? For the voice of the parties, as professional musicians really have to think twice. Good music is left to the people of the value of the United States, not just for music creation and performance of expressive expand the number of vast space.

\section{Representative works and figures}

Wang Jianmin was born in 1956 in Wuxi, Jiangsu Province. He learned music since childhood, and he entered art school to lean musical instruments when he was fifteen-year-old; in 1974, he was admitted to Nanjing Institute of Arts and majored in Composition; in 1982 he was transferred from Wuxi Song and Dance Ensemble to Nanjing Institute of Arts Composition Department, and studied composition theory in Shanghai Conservatory of Music, when he was taught by famous teachs such as Yang Liqing, Lin Hua, Chen Zhonghua and Zhao Xiaosheng, currently he is serving as a teacher at the Shanghai Conservatory of Music. Wang Jianping has created hundreds of works; looking from the learning and working life experience, his creation is obtained through the tireless efforts, the results Fei however, at the same time, exercise his creative grass-roots closer to people's lives, and therefore, his material more from folk music, style more prominent national colors.

His works are of wide range, in which, the influential works include "Tianshan Style" (Erhu and Orchestra), "Four Seasons Glimpse" (Clarinet and piano), "Fantasia" (Zheng solo) and so on. Among them, "Tianshan Style" from its inception in 1993, is widely popular until today have been subjected to different levels of erhu and the audience's favorite, is a good first erhu works. Compared to "First Erhu Rhapsody", "Tianshan Style" is closer to people's aesthetic habits of the public and easy to accept, but "First Erhu Rhapsody" is more for the crowd with professional property professionals, aesthetic concepts, the technical level have shown that age and nationality the new language habits. More prominent features and have the spirit of the times in the works of this period, comes as erhu rhapsody creative works, in addition to "First Erhu Rhapsody" in 2000 and later wrote a series of works "Second Erhu Rhapsody", "Third Erhu Rhapsody", "Third Erhu Rhapsody" and "Fourth Erhu Rhapsody", the influence is more intense.

Liang Yunjiang created more than fifty pieces of works, which are mostly erhu works, including trio "Miao Dance", "Mountain Song", trio "Kangba love", Solo "Mulan Cariccio", double erhu, piano and strings suite "Four seasons." The representative works are erhu concerto "Rivers and Cloud Dream", "Yangtze River" (erhu, piano and cello), "Miluo River" (erhu solo), "Wilderness ditty" (erhu solo) and record issuance. "Chinese Wind" received strong response when performed in Germany and Austria.

"Rivers and Cloud Dream" is one of the masterpieces of modern erhu difficult skills, by professional institutions and bodies young erhu alike. Liangyun Jiang played "River Yunmeng" strong sense of profound mood, with the spirit of the times, its development of the "gravity of bowing," "body movement" and other new playing techniques.

\section{Teaching of erhu concerto and theoretical research}

In 1990s, the study of erhu theory was strengthened, in seminars, while collecting discussion papers composition proceedings, such as: On Hua Yanjun, Liu Tianhua and Hua Yanjun's significant contribution to the development of Chinese erhu music, and so on. Erhu theoretical study of nearly 
500 articles, and is a progressive than 80 years more than 300 articles. From the Chinese Music Collection thesis papers of the more since the founding of the 20th century, the erhu album, theory books, there were 72 before 1980, there were 53 in 1980s, 105 in 1990s, it can also be seen on the erhu Study books increased range of theories.

In 1980s, the textbooks focusing on basic skills training materials erhu were in majority and in 1990s, the theory relatively abundant species. Those focus on style practice include Gao Yaohua's 100 Nanhu Etudes, Wang Guotong's 158 Erhu Style Etudes Those focus on skills include Zhao Hanyang's Erhu pitch and arpeggio exercises, Wang Yongde's Erhu scale arpeggio exercises. Professional textbooks include Wu Suhua's College Erhu learning textbook vol.1,2 and 3. Performing art theory researches: Jiang Fengzhi and Jiang Qing's Jiang Fengzhi Erhu Performing Art, Dong Rongsen's Liu Tianhua Nanhu music research. Erhu Album has been released in different versions in different publishing houses.

In 1990s, subject to market economic impact, erhu popularization became the fashion at this time and to expand the popularity of erhu to social education was an important task of this period. Erhu grading system was implemented in 1990s, and a large number of grading books were published. National Erhu Amateur Grading Portfolio edited by Zhang Shao trial version and official version released a total of five 1-10 set complete set, National Amateur Erhu Grading Works "edited by Xu Jiangde released three times; Chinese Ethnic Orchestral Music Society, National Ethnic Musical Amateur Examination Series - Erhu Album edited by Zhang Shao, Chen Chaoru and others, China Music Society Erhu Amateur Grading Track Selection (Mixed) edited by Committee of erhu Experts and so on.

Zhao Hanyang complied a large number of popular songs for the popularity of the erhu education: 100 Erhu lyric songs, 200 Chinese folks performed by erhu, 100 revolutionary songs performed by erhu, 100 world masterpieces performed by erhu, 100 children songs performed by erhu, Zhao Hanyang and Tang Yitui's 100 Beijing opera melodies performed by erhu; children learn erhu class: Erhu learning guidance for children (Liu Yi'an and Zhao Hanyang), TV Guidance for Children to learn erhu, Introduction for children to learn erhu, Erhu compulsory course, Erhu ensemble practice and so on.

\section{Conclusions}

The development characteristics in the 1990s, generally it belongs to the sluggish development period, the song length partial large-scale works, musical structure with a single movement, multi-movement form. Theme to express personal feelings of the main characteristics of style, personalization is strong. Artificial mode is the new language of expression of this period, irregular rhythm, tonality blur; color has a fantastic sound features. For quick bow technique "difficult" and "odd" to strengthen the full scale exercise, irregular rhythm of practice and other professional and technical training. In the early 1990s, it continued the 1980s pluralism, with respect to the professional nature, in late development into the epidemic, universal characteristics.

\section{References}

[1] Qiao Jianzhong, Yang Guangxiong Chinese music books - Erhu volumn - Instrumental music chapter (Vol.2) Edition, Shanghai Music Publishing House, 2010, 355. 